\section{Internal fixation}

\section{Eithne Comerford}

\section{INTRODUCTION}

The first consideration in fracture planning of a closed fracture is to decide if it is reconstructable (carpentry/ reconstructive) or non-reconstructable (gardening/ biological) which will then direct the methods of internal fixation to be used.

\section{IMPLANT SELECTION}

Always consider the forces that act on your fracture to help deciding on which implants to use. Remember it is a race between the bone healing and the implants failing!

\section{Orthopaedic wires and pins}

Orthopaedic wire is rarely used as the sole method of fixation. It is made of $316 \mathrm{~L}$ stainless steel and sizes range from $0.5 \mathrm{~mm}$ to $1.2 \mathrm{~mm}$. It is used in the following ways: tension, cerclage, hemicerclage and interfragmentary. Cerclage wire is widely misused and associated with high complication rates.

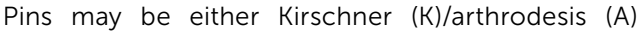
wires or Steinmann pins. K/A wires are essentially mini Steinmann pins $(0.06-1.1 \mathrm{~mm})$ and Steinmann pins are smooth round solid pins (1.6-5.6 mm) (or more). Pins are good at resisting bending but not rotation or compression. An intramedullary (IM) pin can be used as the sole fixation method for stable fractures of the middle third of relatively straight long bones. An IM pin may be combined with other fixation devices to improve the stability of the fixation, for example plate-rod.

\section{Screws and plates}

Screws are distinguished by their thread type and head. They may be self-tapping or non-self-tapping and can have threads within their heads to facilitate placement in a locking plate. Screws can be placed in two separate ways: as positional screws (screw threads gain purchase in all of bone) or as lag screws which will screw bone fragments together. A screw is always placed in lag fashion across a fracture line.

There are several types of plates, for example dynamic compression plates (DCPs), reconstruction plates, T/L plates, acetabular plates. They are made from either $316 \mathrm{~L}$ stainless steel or titanium. Plates have either round, oval (usually non-locking plates) or threaded (locking plates) holes.

Dynamic compression plates (DCPs) have oval screw holes with a sloping shoulder. As the screw is placed eccentrically in the screw hole it slides along the sloping shoulder as it is tightened, compressing the fracture fragments together producing rigid fixation and primary bone healing. The fixation is reliant on the bone/screw/ plate contact and requires accurate reduction and implant contouring.

Locking plates were developed to surmount the limitations of conventional screws and plates as above. They promote a more biological approach to fracture fixation as they do not require completely accurate fracture reduction and implant contouring, leading to less anatomical dissection. Locking plates have either completely threaded hole or combination holes. The fixation relies on the screw head-plate contact rather than the platebone contact.

\section{KEY LEARNING OBJECTIVES}

- Understand the indications for using internal fixation in management of fractures

- Learn about the most common implants used in the internal fixation of fractures and their practical applications

- Understand how fractures heal with different forms of internal fixation and when to become a carpenter (reconstructive approach) or/and a gardener (biological osteosynthesis)

\section{MULTIPLE CHOICE QUESTIONS}

1. Which of the following statements about cerclage wire is correct?
(A) Always used as a method for sole fracture fixation
(B) Rarely associated with complications
(C) Used in short oblique fractures
(D) Commonly applied with a twist fixation

2. Which of the following statements about anatomical reduction and reconstruction of fractures with internal fixation is correct?

(A) Promotes secondary bone healing

(B) Is used in highly comminuted fractures

(C) Is associated with limited soft tissue damage

(D) Is ideal for repairing articular factures

3. What is the appearance of the holes in locking plates?
(A) Oval holes
(B) Threaded holes
(C) Round holes
(D) Square holes 